\title{
Zoonotic potential of Enterocytozoon bieneusi in pre-weaned Korean native calves
}

Sunwoo Hwang ${ }^{1}$, Seung-Uk Shin², SuHee Kim³ ${ }^{3}$ Ji-Hyoung Ryu ${ }^{4}$ and Kyoung-Seong Choi ${ }^{1,2^{*}}$

\begin{abstract}
Background: Enterocytozoon bieneusi is the most common microsporidian species infecting humans and various animals worldwide. To date, there has been limited information on the prevalence of infection and genotypes of E. bieneusi in cattle in the Republic of Korea. Therefore, this study investigated the prevalence and genotypes of $E$. bieneusi circulating in pre-weaned Korean native calves and determined the age pattern of E. bieneusi infection and the relationship between E. bieneusi infection and diarrhea.

Methods: The prevalence of E. bieneusi infection in pre-weaned Korean native calves was screened by polymerase chain reaction. PCR-positive products were sequenced to determine the genotype of E. bieneusi. A Chi-square analysis was used to compare the association between diarrhea and the infection rate of E. bieneusi in each age range or for all ages.

Results: PCR and sequencing analysis revealed an overall prevalence $(16.9 \%, 53 / 314)$ of E. bieneusi in pre-weaned calves. The prevalence of E. bieneusi was highest in September (36.2\%), followed by March (28.3\%). Enterocytozoon bieneusi infection was associated with diarrhea in calves $\left(X^{2}=5.82, P=0.016\right)$. Our results also indicated that $E$. bieneusi infection was significantly associated with calf age $\left(X^{2}=11.61, P=0.003\right)$, and the prevalence of E. bieneusi infection was significantly higher in calves aged 21-40 days-old (odds ratio: 2.90, 95\% confidence interval: 1.54-5.45; $P=0.001)$ than in those aged 1-20 days-old. Interestingly, the association between E. bieneusi infection and diarrhea was observed only in calves aged $1-20$ days-old $\left(x^{2}=5.82, P=0.010\right)$. We identified three known genotypes, BEB4 ( $n$ $=12), \operatorname{BEB} 8(n=21)$ and $\mathrm{J}(n=16)$, and three novel genotypes, BEB8-like $(n=21), \operatorname{KCALF} 1(n=1)$ and KCALF2 $(n=$ 1). The genotype BEB8 was the most prevalent among all age groups. All genotypes identified in this study exhibited zoonotic potential.
\end{abstract}

Conclusions: To our knowledge, this is the first report of the genotype BEB4 in pre-weaned Korean native calves. Zoonotic E. bieneusi infection was prevalent in pre-weaned calves, indicating that cattle may play an important role as a reservoir host for E. bieneusi transmission to humans.

Keywords: Enterocytozoon bieneusi, Pre-weaned Korean native calf, Genotype, Zoonotic infection

\section{Background}

Microsporidia are obligate intracellular protozoanlike fungi that infect a wide range of invertebrates and

\footnotetext{
*Correspondence: kschoi3@knu.ac.kr

${ }^{2}$ Department of Animal Science and Biotechnology, College

of Ecology and Environmental Science, Kyungpook National University, Sangju 37224, Republic of Korea

Full list of author information is available at the end of the article
}

vertebrates including humans [1]. Among approximately 17 human-pathogenic microsporidian species, Enterocytozoon bieneusi is the most common [2]. Enterocytozoon bieneusi usually causes gastrointestinal illnesses such as wasting syndrome and chronic diarrhea in the immunocompromised patients (AIDS or organ transplant recipients, patients with cancer); however, it also leads to asymptomatic and symptomatic infections in 
immunocompetent individuals [3-7]. This species is primarily transmitted through the fecal-oral route, and $E$. bieneusi spores from seemingly healthy animals, humans, and contaminated water or food could be potential sources of infection [8]. Despite the clinical and public health importance of $E$. bieneusi, the implication has not been emphasized because of the low incidence rate in most of the countries.

Genotyping of E. bieneusi and assessment of its host specificity and zoonotic potential are dependent on the sequence analysis of the ribosomal internal transcribed spacer (ITS) $[9,10]$. Currently, 474 E. bieneusi genotypes have been identified in various hosts. In a phylogenetic analysis, the genotypes of $E$. bieneusi have been clustered into at least 11 groups (Groups 1-11) [11]. Group 1 contains the most genotypes found in humans and is considered to be zoonotic. Groups 2-11 have also been found in humans and are associated with various hosts (ruminants, non-human primates, horses, dogs, rabbits, bats, pigs, meerkats, bears, alpacas, chickens and pigeons) [11] and wastewater [12, 13]. To date, more than $50 \mathrm{E}$. bieneusi genotypes have been identified in cattle, most of which belong to Group 2 [14]. Among them, some genotypes (BEB4, BEB6, I and J) were detected in humans [15-18], suggesting that cattle can serve as potential reservoirs of human infection.

According to several studies, BEB4, I and J are common genotypes of $E$. bieneusi found in pre-weaned calves worldwide $[17,19-21]$. However, there is limited information available about the infection rate and genotype distribution of E. bieneusi in pre-weaned Korean native calves. Therefore, this study sought to investigate the prevalence and genotypes of E. bieneusi circulating among pre-weaned Korean native calves, the age pattern of $E$. bieneusi infection, and the relationship between $E$. bieneusi and diarrhea.

\section{Methods}

\section{Sample collection}

From January to October 2018, a total of 314 fecal samples were collected directly by an experienced veterinarian from the rectum of pre-weaned Korean native calves (aged $\leq 60$ days-old) on 10 different farms in the Republic of Korea (ROK), transported to the Animal Immunology Laboratory of Kyungpook National University, ROK, in a cooler with ice packs, and stored at $4{ }^{\circ} \mathrm{C}$ before DNA extraction. The fecal consistency of each calf was categorized as normal or diarrheic according to its physical characteristics.

\section{DNA extraction and PCR amplification}

Genomic DNA was extracted using the QIAamp Fast DNA Stool Mini Kit (Qiagen, Hilden, Germany) using approximately $200 \mathrm{mg}$ of each fecal sample according to the manufacturer's instructions and then stored at $-20{ }^{\circ} \mathrm{C}$ until used in PCR analysis. Enterocytozoon bieneusi was screened based on the ITS region of the rRNA by nested PCR under the following conditions: $94{ }^{\circ} \mathrm{C}$ for $3 \mathrm{~min}$; followed by 35 cycles of $94^{\circ} \mathrm{C}$ for $45 \mathrm{~s}, 55^{\circ} \mathrm{C}$ for 45 $\mathrm{s}$ and $72{ }^{\circ} \mathrm{C}$ for $1 \mathrm{~min}$; and a final extension step at $72{ }^{\circ} \mathrm{C}$ for $10 \mathrm{~min}$ [22]. The amplified fragment was $~ 390 \mathrm{bp}$. Cryptosporidium parvum and Giardia duodenalis infections were also detected using the $60 \mathrm{kDa}$ glycoprotein (gp60) and $\beta$-giardin genes [23-25]. Secondary PCR products were separated by electrophoresis on $1.5 \%$ agarose gels and then visualized after staining with ethidium bromide. In this study, only samples showing a good sequencing result were considered to be positive for $E$. bieneusi.

\section{Sequencing and phylogenetic analysis}

The secondary PCR products were purified using the AccuPower PCR Purification Kit (Bioneer, Daejeon, ROK) and used for direct sequencing (Macrogen, Daejeon, ROK). In order to determine the genotype of $E$. bieneusi, the nucleotide sequences obtained in this study were aligned using ClustalX and were compared with the reference sequences from the GenBank database. A phylogenetic tree was constructed based on the nucleotide alignments using the maximum-likelihood method implemented in the MEGA 7 software [26] and bootstrap analysis was used to evaluate the robustness with 1000 replicates.

\section{Statistical analysis}

Statistical analysis was performed using SPSS Statistics 25 software package for Windows (SPSS Inc., Chicago, IL, USA). Chi-square test was used to compare the association between diarrhea and the infection rate of $E$. bie$n e u s i$ in each age range or all of the ages investigated in this study. In addition, the prevalence of E. bieneusi for each age range was determined using binary univariate logistic regression models. The odds ratio (OR) and 95\% confidence intervals $(\mathrm{CI})$ were calculated to determine the likelihood of association. A $P$-value of $\leq 0.05$ was considered to be statistically significant.

\section{Results \\ Prevalence of $E$. bieneusi}

The overall prevalence of E. bieneusi was found to be $16.9 \%(53 / 314)$ in pre-weaned Korean native calves regardless of diarrhea. Among the 10 different farms examined, $E$. bieneusi was detected in 6 farms (Table 1). We compared the infection rate of E. bieneusi according to the month of sampling. As shown in Table 2, the prevalence of E. bieneusi was highest in September 
Table 1 Prevalence and genotypes of E. bieneusi identified in pre-weaned Korean native calves

\begin{tabular}{|c|c|c|c|}
\hline Region & N & No. of positive samples & ITS genotype \\
\hline Anseong & 39 & 4 & $\operatorname{BEB} 8(n=2), J(n=2)$ \\
\hline Geochang & 78 & 22 & $\operatorname{BEB} 4(n=3), \operatorname{BEB} 8(n=7), \operatorname{BEB} 8-$ like $(n=1), \mathrm{J}(n=11)$ \\
\hline Gimje & 71 & 1 & $\operatorname{KCALF}^{\mathrm{a}}(n=1)$ \\
\hline Gyeongju & 6 & 0 & - \\
\hline Jeongeup & 1 & 0 & - \\
\hline Mungyeong & 82 & 21 & $\operatorname{BEB} 4(n=9), \operatorname{BEB} 8(n=8), \operatorname{BEB} 8-\operatorname{lik}^{\mathrm{a}}(n=1), J(n=2), \operatorname{KCALF}^{\mathrm{a}}(n=1)$ \\
\hline Naju & 1 & 0 & - \\
\hline Sangju & 2 & 1 & $J(n=1)$ \\
\hline Yechoen & 1 & 0 & - \\
\hline Youngju & 33 & 4 & $\operatorname{BEB} 8(n=4)$ \\
\hline Total & 314 & 53 & $\begin{array}{l}\operatorname{BEB} 4(n=12), \operatorname{BEB} 8(n=21), \operatorname{BEB}^{2}-\text { like }^{\mathrm{a}}(n=2), \mathrm{J}(n=16), \operatorname{KCALF}^{\mathrm{a}}(n \\
\quad=1), \operatorname{KCALF}^{\mathrm{a}}(n=1)\end{array}$ \\
\hline
\end{tabular}

${ }^{a}$ Novel genotypes found in this study

Abbreviation: $N$, number of calves examined

Table 2 Prevalence of E. bieneusi in pre-weaned Korean native calves according to the month

\begin{tabular}{lll}
\hline Month & No. positive/No. examined & Prevalence (\%) \\
\hline January & $0 / 2$ & 0 \\
March & $15 / 53$ & 28.3 \\
April & $6 / 68$ & 8.8 \\
May & $3 / 27$ & 11.1 \\
June & $1 / 23$ & 4.3 \\
July & $0 / 12$ & 0 \\
August & $0 / 7$ & 0 \\
September & $17 / 47$ & 36.2 \\
October & $11 / 75$ & 14.7 \\
Total & $53 / 314$ & 16.9 \\
\hline
\end{tabular}

(36.2\%), followed by March (28.3\%) and October (14.7\%); however, $E$. bieneusi infection was not detected in July and August. When E. bieneusi infection was compared according to the fecal consistency, there were $11.9 \%$ and $22.1 \%$ infection rates in both diarrheic and normal feces, respectively. Co-infection with $E$. bieneusi and $C$. parvum was not detected; however, co-infection with E. bieneusi and G. duodenalis was observed in diarrheic $(6.3 \%, 10 / 314)$ and normal feces $(1.9 \%, 3 / 314)$. Although there was no statistically significant difference, the risk of diarrhea was increased by 3.36-fold during co-infection with E. bieneusi and G. duodenalis (95\% CI: 0.91-12.43, $P=0.056$; Table 3). Enterocytozoon bieneusi infection was associated with diarrhea $\left(\chi^{2}=5.82, d f=1, P=0.016\right.$; Table 4). Enterocytozoon bieneusi-positive samples were compared according to the age group of the calves. As shown in Table 4, the prevalence of E. bieneusi was the highest in calves aged 21-40 days-old, followed by those aged 41-60 days-old and 1-20 days-old $\left(\chi^{2}=11.61, d f=\right.$ 2, $P=0.003$ ). The risk of being positive to $E$. bieneusi was 2.9-fold higher in calves aged 21-40 days-old (95\% CI: $1.54-5.45, P=0.001)$ than in those aged 1-20 days-old. The association between E. bieneusi infection and diarrhea according to the age group was analyzed by the Chisquare test. Enterocytozoon bieneusi infection was found to be associated with diarrhea only in calves aged 1-20 days-old $\left(\chi^{2}=6.61, d f=1, P=0.010\right.$; Table 5).

\section{Genotypes of E. bieneusi}

To determine the genotypes of E. bieneusi detected in pre-weaned Korean native calves, a total of 53 ITS-positive samples were sequenced. The length of a $243 \mathrm{bp}$ sequence was used to construct the phylogenetic tree.

Table 3 Detection rates of all pathogen species in pre-weaned Korean native calves according to the diarrhea status

\begin{tabular}{lllll}
\hline Pathogen & $\begin{array}{l}\text { Positive in diarrhea samples } \\
(n=160)\end{array}$ & $\begin{array}{l}\text { Positive in non-diarrhea samples } \\
(n=154)\end{array}$ & $x^{2}(P$-value $)$ & OR (95\% Cl) \\
\hline Cryptosporidium parvum & $11(6.9 \%)$ & $14(9.1 \%)$ & $0.53(0.468)$ & $0.74(0.32-1.68)$ \\
Giardia duodenalis & $23(14.4 \%)$ & $18(11.7 \%)$ & $0.50(0.480)$ & $1.27(0.66-2.46)$ \\
Enterocytozoon bieneusi & $19(11.9 \%)$ & $34(22.1 \%)$ & $5.82(0.016)^{*}$ & $0.48(0.26-0.88)$ \\
E. bieneusi + G. duodenalis & $10(6.3 \%)$ & $3(1.9 \%)$ & $3.66(0.056)$ & $3.36(0.91-12.43)$ \\
\hline
\end{tabular}

${ }^{*} P<0.05$ 
Table 4 Association between physical variables and E. bieneusi infection in pre-weaned Korean native calves

\begin{tabular}{llll}
\hline Variable & $\begin{array}{l}\text { Frequency of } E . \\
\text { bieneusi positivity } \\
(\%)\end{array}$ & $x^{2}(P$ value $)$ & OR $(95 \% \mathrm{Cl})$ \\
\hline $\begin{array}{l}\text { Fecal consistency } \\
\begin{array}{c}\text { Non-diarrhea } \\
\text { (Ref.) }\end{array}\end{array}$ & $34 / 154(22.1)$ & $5.82(0.016)^{*}$ & 1.00 \\
$\begin{array}{l}\text { Diarrhea } \\
\text { Age (days) }\end{array}$ & $19 / 160(11.9)$ & & $0.48(0.26-0.88)^{*}$ \\
$1-20$ (Ref.) & $19 / 173(11.0)$ & $11.61(0.003)^{* *}$ & 1.00 \\
$21-40$ & $30 / 114(26.3)$ & & $2.90(1.54-5.45)^{* *}$ \\
$41-60$ & $4 / 17(14.8)$ & & $1.41(0.44-4.51)$ \\
\hline
\end{tabular}

${ }^{*} P<0.05,{ }^{* *} P<0.005$ vs reference (Ref.)

Three distinct genotypes, BEB4 $(n=12$, identical to GenBank: KF675194), BEB8 $(n=21$, identical to GenBank: KT984487) and J ( $n=16$, identical to GenBank: MN178156) were found. Moreover, three novel genotypes, BEB8-like $(n=2), \operatorname{KCALF1}(n=1)$ and KCALF2 $(n=1)$, were identified. Of the 53 ITS-positive samples, 16 sequences were included in the phylogenetic tree, and all of the genotypes identified in this study belonged to the zoonotic Group 2 (Fig. 1). Among them, BEB8 was found to be the most prevalent genotype in pre-weaned calves regardless of diarrhea. In contrast, the genotypes KCALF1 and KCALF2 were found only in one calf aged 16 days and 10 days with diarrhea, respectively. In particular, the genotypes BEB8 and J were detected in all age groups (Table 6). BEB4 was found only in calves aged up to 40 days-old and only on two farms. Calves aged 1-20 days-old had a higher diversity of genotypes, with six genotypes being found and the diversity of genotypes decreased with age (Table 6). The BEB8-like genotype showed only one nucleotide difference from the genotype BEB8. The novel genotype KCALF1 differed by four nucleotides relative to CHN6 (GenBank: MN136773), which was found in the feces of humans in China. KCALF2 also showed four nucleotide differences from genotype I (GenBank: MT231513) isolated from cattle feces. As shown in Table 1, the distribution of E. bieneusi genotypes on the farms was different. Three farms (Gimje, Sangju and Yeongju) had only one genotype, whereas the others had two to five genotypes (Table 1). To the best of our knowledge, this is the first report of the presence of the genotype BEB4 in pre-weaned Korean native calves.

\section{Discussion}

The present study showed that the infection rate of E. bieneusi in pre-weaned Korean native calves was $16.9 \%$, which is similar to that reported in other studies for cattle in the ROK and several other countries [17, 27-30]. The prevalence of E. bieneusi in preweaned calves varied from $0 \%$ to $50 \%$ depending on the farm. This variation could be attributed to nutrition, herd management practices, health of the animal, and hygiene. Interestingly, E. bieneusi infection appears to be closely related to seasonal differences. According to our results, E. bieneusi infection tended to occur primarily in September (36.2\%) and March (28.3\%); in contrast, the incidence rate of E. bieneusi was rather low in warmer seasons with no infections in July and August. This result is different from that of a previous study reporting a higher prevalence during warmer seasons in the ROK [27]. The differences between these two studies might be explained by the difference in the number of samples collected each month and the age of the calf. However, our results were fairly consistent with those of a study conducted in China, which reported a higher prevalence in spring [31]. Although the results are inconclusive, the transmission of $E$. bieneusi may be related to seasonal variations. Thus, further studies are required to investigate the association between $E$. bieneusi infection and seasonal variations.

In this study, according to the Chi-square analysis, $E$. bieneusi infection was associated with diarrhea; however, the infection rate was not high in diarrheic feces. Although E. bieneusi was detected in diarrheic feces, it

Table 5 Association between diarrhea and presence of E. bieneusi in pre-weaned Korean native calves according to age

\begin{tabular}{|c|c|c|c|c|c|}
\hline Age (days) & Fecal consistency & $\begin{array}{l}\text { Frequency of E. bieneusi } \\
\text { positivity (\%) }\end{array}$ & $x^{2}(P$-value $)$ & OR & $95 \% \mathrm{Cl}$ \\
\hline \multirow[t]{2}{*}{$1-20$} & Diarrhea & $6 / 102(5.9)$ & $6.61(0.010)^{*}$ & 0.28 & $0.10-0.77$ \\
\hline & Non-diarrhea & 13/71 (18.3) & & & \\
\hline \multirow[t]{2}{*}{$21-40$} & Diarrhea & $10 / 44(22.7)$ & $0.48(0.490)$ & 0.74 & $0.31-1.76$ \\
\hline & Non-diarrhea & 20/70 (28.6) & & & \\
\hline \multirow[t]{2}{*}{$41-60$} & Diarrhea & $3 / 14(21.4)$ & $1.01(0.596)$ & 3.27 & $0.30-36.31$ \\
\hline & Non-diarrhea & $1 / 13(7.7)$ & & & \\
\hline
\end{tabular}

${ }^{*} P<0.05$ vs reference (Ref.) 


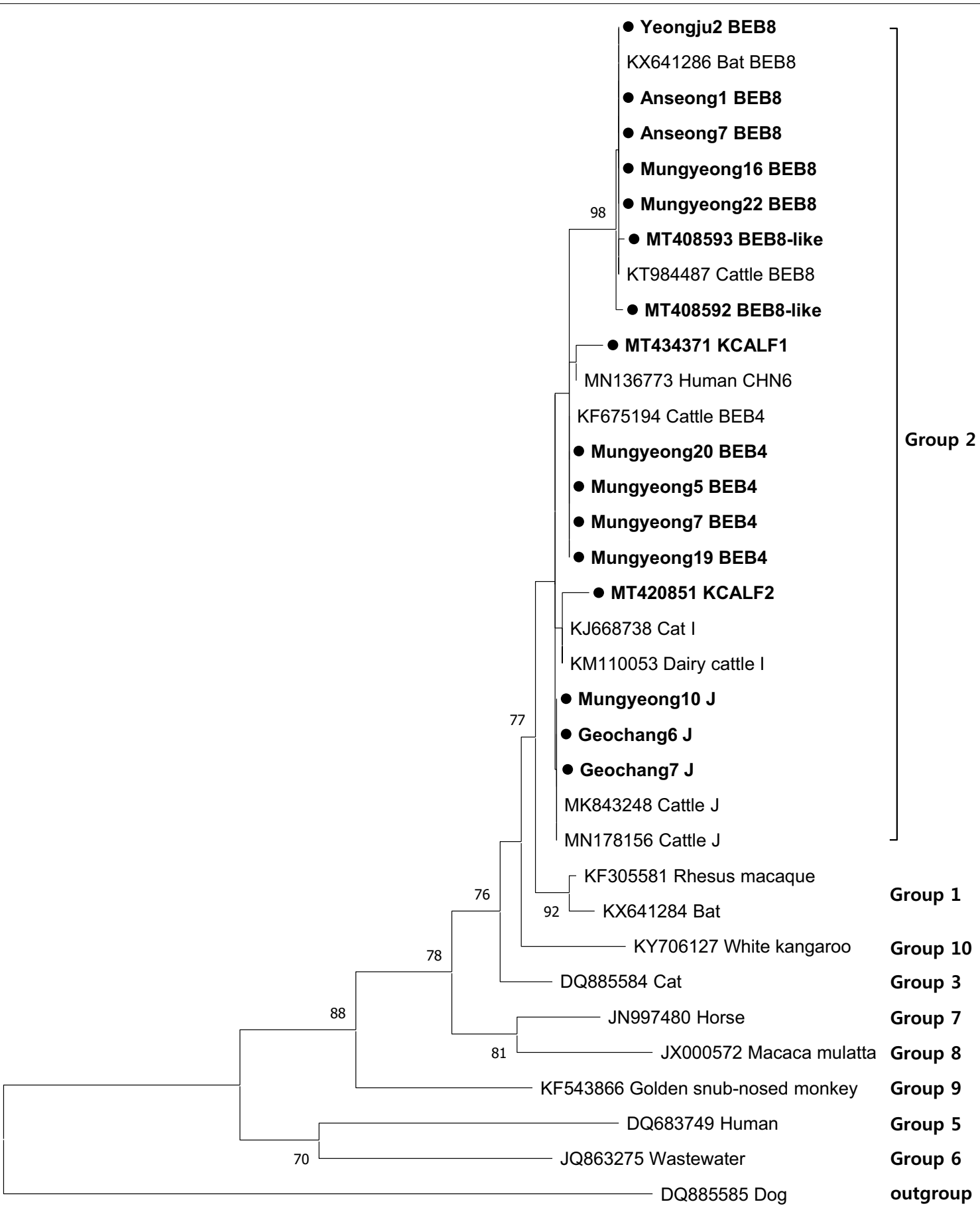

Fig. 1 Phylogenetic relationships of Enterocytozoon bieneusi genotypes identified in this study and other reported genotypes based on the 243 bp internal transcribed spacer gene sequences. The tree was constructed using MEGA 7 software with the maximum-likelihood method. Numbers at the nodes of the tree indicate bootstrap values as a percentage of 1000 replicates that support each phylogenetic branch. The isolates identified in this study are marked in bold type with a circle symbol

is unlikely that $E$. bieneusi is associated with diarrhea in pre-weaned calves (OR $=0.48,95 \% \mathrm{CI}: 0.26-0.88, P=$ 0.016 ; Table 3). However, a previous study performed in
China revealed that E. bieneusi infection increased 2.5fold in pre-weaned calves with diarrhea (95\% CI: 1.7-3.8, $P \leq 0.0001)$ compared with those without diarrhea, and 
Table 6 Genotype distribution of E. bieneusi according to age in pre-weaned Korean native calves

\begin{tabular}{|c|c|c|c|c|c|c|c|}
\hline Age (days) & BEB4 & BEB8 & BEB8-like & J & KCALF1 & KCALF2 & Total \\
\hline $1-20$ & 4 & 7 & 1 & 5 & 1 & 1 & 22 \\
\hline $21-40$ & 8 & 11 & 1 & 10 & - & - & 30 \\
\hline $41-60$ & - & 3 & - & 1 & - & - & 4 \\
\hline Total & 12 & 21 & 2 & 16 & 1 & 1 & 53 \\
\hline
\end{tabular}

most of all, E. bieneusi infection was associated with diarrhea [32]. The difference between the two groups could be explained by the number of samples and the age of the calf. Thus, the relationship between E. bieneusi infection and diarrhea should be determined through further investigation.

Interestingly, among the three pathogens examined, the infection rate of $E$. bieneusi was the highest in preweaned Korean native calves. This might have been overlooked in diagnostic tests due to uncertainty regarding the role of $E$. bieneusi as a pathogen in calf diarrhea. Furthermore, it is possible that the significance of E. bieneusi has not been prominently recognized in the field. Results of the present study showed that co-infection with E. bieneusi and G. duodenalis was not statistically significant $(P$ $=0.056$; Table 3); however, it was 3.36-fold more likely to cause diarrhea (95\% CI: 0.91-12.43) compared with a single infection with $E$. bieneusi in calves. Our result was inconsistent with that reported in China; co-infection with $E$. bieneusi and G. duodenalis was significantly associated with diarrhea [32]. In this study, the number of positive samples co-infected with two pathogens was small; thus, these results failed to demonstrate an association between diarrhea and co-infection in pre-weaned calves. Although it remains unclear, such co-infection might increase the severity and duration of diarrhea in calves. More epidemiological investigations are required to determine whether the occurrence of diarrhea is more common in calves co-infected with E. bieneusi and G. duodenalis.

The prevalence of $E$. bieneusi in calves was significantly associated with the age of the calf. The infection rate of $E$. bieneusi was the highest in calves aged 21-40 days-old, followed by calves aged 41-60 days-old, and 1-20 daysold. In comparison with calves aged $\leq 20$ days-old, the risk of E. bieneusi infection was 2.9-fold higher in calves aged $21-40$ days-old ( $P=0.003$; Table 4$)$. A possible explanation is that the immune status of calves in this age group may result in a higher susceptibility to E. bieneusi infection due to the loss of the maternal antibodies [33]. To date, several studies have demonstrated age-related prevalence patterns of E. bieneusi infection [19, 28, 29, 34]. However, in contrast to our findings, the prevalence of $E$. bieneusi has been found to increase with age [19, 35,
36]. In the ROK, there are limited studies on E. bieneusi infection in cattle; thus, it is not possible to compare the prevalence of $E$. bieneusi according to age group. In addition, there is no information on the transmission route of E. bieneusi on the farms examined; however, E. bieneusi infection may be related to the hygiene status of farms rather than the calf age. Therefore, to prevent $E$. bieneusi infection, the farming management system should be improved, which could include no contact with contaminated food and water, cleaning, and disinfection.

We also investigated the association between E. bieneusi infection and diarrhea according to the age group. Our results revealed that there was a significant correlation between $E$. bieneusi infection and diarrhea in calves aged 1-20 days-old ( $P=0.010$; Table 5$)$. However, this has been shown to be associated with a low incidence of diarrhea in E. bieneusi-infected calves. Based on the results, it is unlikely that E. bieneusi is the primary pathogen that causes diarrhea in pre-weaned Korean native calves. Cattle may be a source of environmental contamination by $E$. bieneusi. Therefore, E. bieneusi infection in calves should be considered as a zoonotic potential rather than a causative agent of diarrhea.

In the present study, sequence analysis of the ITS gene from $53 \mathrm{E}$. bieneusi-positive isolates identified six genotypes (BEB4, BEB8, J, BEB8-like, KCALF1 and KCALF2) belonging to zoonotic Group 2. Unlike previous studies, the genotype BEB8 was the most prevalent in pre-weaned Korean native calves and was found in $45.3 \%(24 / 53)$ of the positive samples. Moreover, this genotype was commonly identified in all age groups regardless of diarrhea. Several studies have reported that the genotype BEB8 can be found not only in cattle [29, 37, 38] but also in bats [39] and rabbits [40], indicating that this genotype might have a potential risk for zoonotic infection in humans. Genotype J, identified in $30.2 \%(16 / 53)$ of E. bieneusi-positive samples, was the second most common genotype in all age groups. BEB4 was the third most common genotype and found in calves only up to 40 days-old. BEB4 has been identified as a zoonotic genotype in cattle in many countries; however, it was first detected in the ROK. Interestingly, genotype I, with a wide range of hosts, was not detected in this study. In contrast, a previous study conducted in the ROK reported the presence of genotype 
I in three cattle [26]. This can be attributed to the low incidence of genotype I in cattle in the ROK compared to that of other countries. The present study reported the identification of three novel genotypes in pre-weaned Korean native calves, indicating that high genetic diversity exists in the E. bieneusi ITS region. Furthermore, the genotypes BEB4, BEB8 and J were common genotypes in pre-weaned Korean native calves. The differences in the distribution of E. bieneusi in pre-weaned calves according to farms may be attributed to the geographical location and the farm management system. All genotypes identified in this study have a possible zoonotic potential, suggesting that cattle play an important role as a reservoir host in E. bieneusi transmission to humans.

\section{Conclusions}

This study evaluated the presence and genotypes of $E$. bieneusi detected in pre-weaned Korean native calves. Enterocytozoon bieneusi infection was associated with diarrhea in calves aged 1-20 days-old, and the prevalence of $E$. bieneusi was significantly higher in calves aged 21-40 days-old. ITS sequencing identified six genotypes (BEB4, BEB8, J, BEB8-like, KCALF1 and KCALF2), with BEB8 being the most prevalent genotype in pre-weaned Korean native calves. The identification of zoonotic genotypes in pre-weaned calves suggests that these animals could play an important role as reservoir hosts for zoonotic infections.

\section{Abbreviations \\ Cl: confidence interval; ITS: internal transcribed spacer; OR: odds ratio; PCR: polymerase chain reaction; ROK: Republic of Korea.}

\section{Acknowledgements}

We thank Jeong-Byoung Chae, DVM for collecting feces and Yoon-Joo Lee for helping with DNA preparation.

\section{Authors' contributions \\ KSC designed the research and wrote the manuscript. SWH, SUS and JHR performed the experiments. SHK analyzed the data. All authors read and approved the final manuscript.}

\section{Funding}

This study was supported by the National Research Foundation of Korea (NRF), funded by the Korea government (MSIP) (No. 2018R1D1A1B07048271).

\section{Availability of data and materials}

All data generated or analyzed during this study are included in the article. The nucleotide sequences obtained in the present study have been deposited in the GenBank database under the accession numbers MT408592-MT408593 for genotype BEB8-like, MT434371 for genotype KCALF1, and MT420851 for genotype KCALF2.

\section{Ethics approval and consent to participate}

This study did not receive approval from the Institutional Animal Care and Use Committee (IACUC) at Kyungpook National University because the IACUC at this University evaluates laboratory animals maintained within indoor facilities and not outdoor animals. A local veterinarian collected all of the fecal samples from the animals on different farms and sent them to the researchers.
All procedures and possible consequences were explained to farm owners/ managers of the surveyed farms. Written informed consent was obtained for the collection of fecal samples from the owners of the Korean native calves. Permission was also obtained from farm owners/managers prior to the collection of fecal samples.

\section{Consent for publication}

Not applicable.

\section{Competing interests}

The authors declare that they have no competing interests.

\section{Author details}

${ }^{1}$ Department of Horse/Companion and Wild Animal Science, College of Ecology and Environmental Science, Kyungpook National University, Sangju 37224, Republic of Korea. ${ }^{2}$ Department of Animal Science and Biotechnology, College of Ecology and Environmental Science, Kyungpook National University, Sangju 37224, Republic of Korea. ${ }^{3}$ Gyeongsang National University Hospital, Jinju 52727, Republic of Korea. ${ }^{4}$ Foreign Animal Disease Division, Animal and Plant Quarantine Agency, Gimcheon 39660, Republic of Korea.

Received: 13 April 2020 Accepted: 5 June 2020

Published online: 10 June 2020

\section{References}

1. Stentiford GD, Becnel J, Weiss LM, Keeling PJ, Didier ES, Williams BP, et al. Microsporidia_emergent pathogens in the global food chain. Trends Parasitol. 2016;32:336-48.

2. Didier ES, Weiss LM. Microsporidiosis: current status. Curr Opin Infect Dis. 2006;19:485-92.

3. Didier ES, Weiss LM. Microsporidiosis: not just in AIDS patients. Curr Opin Infect Dis. 2011;24:490-5.

4. Galvan AL, Sanchez AM, Valentin MA, Henriques-Gil N, Izquierdo F, Fenoy $\mathrm{S}$, et al. First cases of microsporidiosis in transplant recipients in Spain and review of the literature. J Clin Microbiol. 2011;49:1301-6.

5. Matos O, Lobo ML, Xiao L. Epidemiology of Enterocytozoon bieneusi infection in humans. J Parasitol Res. 2012;2012:981424.

6. Nkinin SW, Asonganyi T, Didier ES, Kaneshiro ES. Microsporidian infection is prevalent in healthy people in Cameroon. J Clin Microbiol. 2007:45:2841-6.

7. Sak B, Kvac M, Kucerova Z, Kvetonova D, Sakova K. Latent microsporidial infection in immunocompetent individuals—a longitudinal study. PLoS Negl Trop Dis. 2011;5:e1162

8. Chen D, Wang SS, Zou Y, Li Z, Xie SC, Shi LQ, et al. Prevalence and multi-locus genotypes of Enterocytozoon bieneusi in black-boned sheep and goats in Yunnan Province, southwestern China. Infect Genet Evol. 2018;65:385-91.

9. Santin M, Fayer R. Microsporidiosis: Enterocytozoon bieneusi in domesticated and wild animals. Res Vet Sci. 2011;90:363-71.

10. Santin M, Fayer R. Enterocytozoon bieneusi genotype nomenclature based on the internal transcribed spacer sequence: a consensus. J Eukaryot Microbiol. 2009;56:34-8.

11. LiW, Feng Y, Santin M. Host specificity of Enterocytozoon bieneusi and public health implications. Trends Parasitol. 2019;35:436-51.

12. Guo Y, Alderisio KA, Yang W, Cama V, Feng Y, Xiao L. Host specificity and source of Enterocytozoon bieneusi genotypes in a drinking source watershed. Appl Environ Microbiol. 2014;80:218-25.

13. Yu F, Qi M, Zhao Z, Lv C, Wang Y, Wang R, et al. The potential role of synanthropic rodents and flies in the transmission of Enterocytozoon bieneusi on a dairy cattle farm in China. J Eukaryot Microbiol. 2019;66:435-41.

14. Zhang Y, Koehler AV, Wang T, Haydon SR, Gasser RB. Enterocytozoon bieneusi genotypes in cattle on farms located within a water catchment area. J Eukaryot Microbiol. 2019;66:553-9.

15. Li N, Xiao L, Wang L, Zhao S, Zhao X, Duan L, et al. Molecular surveillance of Cryptosporidium spp., Giardia duodenalis, and Enterocytozoon bieneusi by genotyping and subtyping parasites in wastewater. PLoS Negl Trop Dis. 2012;6:e1809.

16. Zhao W, Wang J, Yang Z, Liu A. Dominance of the Enterocytozoon bieneusi genotype BEB6 in red deer (Cervus elaphus) and siberian roe 
deer (Capreolus pygargus) in China and a brief literature review. Parasite. 2017:24:54.

17. Del Coco VF, Cordoba MA, Bilbao G, de Almeida Castro P, Basualdo JA, Santin M. First report of Enterocytozoon bieneusi from dairy cattle in Argentina. Vet Parasitol. 2014;199:112-5.

18. Zhang $X$, Wang $Z$, Su Y, Liang $X$, Sun $X$, Peng $S$, et al. Identification and genotyping of Enterocytozoon bieneusi in China. J Clin Microbiol. 2011:49:2006-8

19. Santin M, Fayer R. A longitudinal study of Enterocytozoon bieneusi in dairy cattle. Parasitol Res. 2009;105:141-4.

20. Jiang Y, Tao W, Wan Q, Li Q, Yang Y, Lin Y, et al. Zoonotic and potentially host-adapted Enterocytozoon bieneusi genotypes in sheep and cattle in northeast China and an increasing concern about the zoonotic importance of previously considered ruminant-adapted genotypes. Appl Environ Microbiol. 2015;81:3326-35.

21. Fayer R, Santin M, Macarisin D. Detection of concurrent infection of dairy cattle with Blastocystis, Cryptosporidium, Giardia, and Enterocytozoon by molecular and microscopic methods. Parasitol Res. 2012:111:1349-55.

22. Sulaiman IM, Fayer R, Lal AA, Trout JM, Schaefer FW 3rd, Xiao L. Molecular characterization of microsporidia indicates that wild mammals harbor host-adapted Enterocytozoon spp. as well as human-pathogenic Enterocytozoon bieneusi. Appl Environ Microbiol. 2003;69:4495-501.

23. Caccio SM, De Giacomo M, Pozio E. Sequence analysis of the beta-giardin gene and development of a polymerase chain reaction-restriction fragment length polymorphism assay to genotype Giardia duodenalis cysts from human faecal samples. Int J Parasitol. 2002;32:1023-30.

24. Lalle M, Pozio E, Capelli G, Bruschi F, Crotti D, Caccio SM. Genetic heterogeneity at the beta-giardin locus among human and animal isolates of Giardia duodenalis and identification of potentially zoonotic subgenotypes. Int J Parasitol. 2005;35:207-13.

25. Alves M, Xiao L, Sulaiman I, Lal AA, Matos O, Antunes F. Subgenotype analysis of Cryptosporidium isolates from humans, cattle, and zoo ruminants in Portugal. J Clin Microbiol. 2003:41:2744-7.

26. Kumar S, Stecher G, Tamura K. MEGA7: Molecular evolutionary genetics analysis version 7.0 for bigger datasets. Mol Biol Evol. 2016;33:1870-4.

27. Lee JH. Prevalence and molecular characteristics of Enterocytozoon bieneusi in cattle in Korea. Parasitol Res. 2007;101:391-6.

28. Jurankova J, Kamler M, Kovarcik K, Koudela B. Enterocytozoon bieneusi in bovine viral diarrhea virus (BVDV) infected and noninfected cattle herds. Res Vet Sci. 2013;94:100-4.
29. da Silva Fiuza VR, Lopes CW, de Oliveira FC, Fayer R, Santin M. New findings of Enterocytozoon bieneusi in beef and dairy cattle in Brazil. Vet Parasitol. 2016;216:46-51.

30. Qi M, Jing B, Jian F, Wang R, Zhang S, Wang H, et al. Dominance of Enterocytozoon bieneusi genotype $\mathrm{J}$ in dairy calves in Xinjiang, northwest China. Parasitol Int. 2017;66:960-3.

31. Feng Y, Gong X, Zhu K, Li N, Yu Z, Guo Y, et al. Prevalence and genotypic identification of Cryptosporidium spp., Giardia duodenalis and Enterocytozoon bieneusi in pre-weaned dairy calves in Guangdong, China. Parasit Vectors. 2019;12:41.

32. Wang R, Li N, Jiang W, Guo Y, Wang X, Jin Y, et al. Infection patterns, clinical significance, and genetic characteristics of Enterocytozoon bieneusi and Giardia duodenalis in dairy cattle in Jiangsu, China. Parasitol Res. 2019;118:3053-60.

33. Zhao W, Zhang W, Yang F, Zhang L, Wang R, Cao J, et al. Enterocytozoon bieneusi in dairy cattle in the northeast of China: genetic diversity of ITS gene and evaluation of zoonotic transmission potential. J Eukaryot Microbiol. 2015;62:553-60.

34. Tang C, Cai M, Wang L, Guo Y, Li N, Feng Y, et al. Genetic diversity within dominant Enterocytozoon bieneusi genotypes in pre-weaned calves. Parasit Vectors. 2018;11:170.

35. Fayer R, Santin M, Trout JM. First detection of microsporidia in dairy calves in North America. Parasitol Res. 2003;90:383-6.

36. Fayer R, Santin M, Trout JM. Enterocytozoon bieneusi in mature dairy cattle on farms in the eastern United States. Parasitol Res. 2007;102:15-20.

37. Santin M, Dargatz D, Fayer R. Prevalence and genotypes of Enterocytozoon bieneusi in weaned beef calves on cow-calf operations in the USA. Parasitol Res. 2012;110:2033-41.

38. Li J, Luo N, Wang C, Qi M, Cao J, Cui Z, et al. Occurrence, molecular characterization and predominant genotypes of Enterocytozoon bieneusi in dairy cattle in Henan and Ningxia, China. Parasit Vectors. 2016:9:142.

39. Lee SH, Oem JK, Lee SM, Son K, Jo SD, Kwak D. Molecular detection of Enterocytozoon bieneusi from bats in South Korea. Med Mycol. 2018;56:1033-7.

40. Zhang X, Qi M, Jing B, Yu F, Wu Y, Chang Y, et al. Molecular characterization of Cryptosporidium spp., Giardia duodenalis, and Enterocytozoon bieneusi in rabbits in Xinjiang, China. J Eukaryot Microbiol. 2018;65:854-9.

\section{Publisher's Note}

Springer Nature remains neutral with regard to jurisdictional claims in published maps and institutional affiliations.
Ready to submit your research? Choose BMC and benefit from:

- fast, convenient online submission

- thorough peer review by experienced researchers in your field

- rapid publication on acceptance

- support for research data, including large and complex data types

- gold Open Access which fosters wider collaboration and increased citations

- maximum visibility for your research: over $100 \mathrm{M}$ website views per year

At BMC, research is always in progress.

Learn more biomedcentral.com/submissions 